\title{
Diagnosis and Treatment Options for Pulmonary Embolism (PE) in the Acute Care Setting
}

\author{
Simona Campa, Raymund Gantioque \\ California State University, Los Angeles, CA, USA \\ Email: scampa5@calstatela.edu
}

How to cite this paper: Campa, S. and Gantioque, R. (2018) Diagnosis and Treatment Options for Pulmonary Embolism (PE) in the Acute Care Setting. World Journal of Cardiovascular Diseases, 8, 152-161. https://doi.org/10.4236/wjcd.2018.82015

Received: January 11, 2018

Accepted: February 10, 2018

Published: February 13, 2018

Copyright $\odot 2018$ by authors and Scientific Research Publishing Inc. This work is licensed under the Creative Commons Attribution International License (CC BY 4.0).

http://creativecommons.org/licenses/by/4.0/

\section{cc) (i) Open Access}

\begin{abstract}
Thromboembolic diseases continue to be one of the most prevalent medical problems today and can lead to life-threatening conditions, such as pulmonary embolism (PE). Currently, PE diagnosis and treatment are a challenge because of acute onset right ventricular strain with right-sided heart failure, sudden death, pulmonary infarction, and cardiogenic shock, which limit the time for therapeutic success. The aim of this study was to evaluate our perception, knowledge, and concerns regarding PE, discuss the importance of promptly diagnosing PE to provide appropriate treatment options for this life-threatening condition, list the most common clinical manifestations present when PE is suspected, and review the clinical approach to patients with suspected PE in an inpatient setting. In addition, this study reviews the risk stratification of patients with PE and treatment options beyond anticoagulation, compares new treatment options for patients presenting with acute symptomatic PE, and compares aspiration catheters (10 F Pronto .035" and 14 F XL extraction catheter (Vascular Solutions, Minneapolis, MN)) and ultrasound-assisted catheter-directed thrombolysis (USAT) versus systemic thrombolysis. This literature review was limited by the quality and number of studies available regarding new treatment options for patients presenting with acute symptomatic PE. Thus, more studies are needed to prove the validity of newer treatment options being trialed, such as aspiration catheters $(10 \mathrm{~F}$ Pronto .035" and 14 F XL extraction catheter) and USAT, with the hope that further studies will guide patient management and increase our understanding of next generation aspiration catheters, which may provide novel insights on treating acute symptomatic PE.
\end{abstract}

\section{Keywords}

Pulmonary Embolism (PE), Aspiration Catheters (The 10 F Pronto .035" and 
14 F XL Extraction Catheter (Vascular Solutions, Minneapolis, MN)), The Ultrasound-Assisted Catheter-Directed Thrombolysis (USAT), Systemic Thrombolysis

\section{Introduction}

According to Goldman and Schafer [1], pulmonary embolism (PE) is an obstruction of a pulmonary artery by material that has traveled to the lungs through the bloodstream. The most common type of material that travels to the lungs is a thrombus from the legs or arms and is known as venous thromboembolism (VTE). Fat from bone marrow that might form after a fracture, air injected during intravenous infusion, tumor cells, and certain medical equipment are also examples of possible causes that can lead to blood flow obstruction.

The blockage results in right ventricular strain and pulmonary hypertension, which cause the majority of PE symptoms. These symptoms are related to impaired gas exchange due to alveolar surface loss and reflex bronchoconstriction [1].

Approximately 50,000 deaths per year occur in the United States due to PE [2].

\section{Epidemiology}

Despite advances in the past 30 years, PE continues to have a high mortality rate, and diagnosis and treatment have not changed significantly in the last decade [3].

VTE, which includes deep vein thrombosis (DVT) and PE, is considered the third most common cause of cardiovascular death after stroke and myocardial infarction [2]. If not treated promptly, acute PE has a mortality rate as high as $30 \%$ with a death rate of $8 \%$ [3].

Goldman and Schafer [1] postulate that the incidence of VTE increases exponentially with age and that there higher incidence in African-Americans and Caucasian-Americans than in Asian Pacific Islanders and the Hispanic population. Moreover, most studies suggest that African-Americans have higher rates of VTE than Caucasian-Americans possibly due to risk factors, such as diabetes, obesity, and elevated factor VIII [4].

In 2014, Zakai et al. [4] examined the association of race with VTE in over 438,090 people in three prospective studies; the Cardiovascular Health Study (CHS), the Atherosclerosis Risk in Communities study (ARIC), and the reasons for Geographic and Racial Differences in Stroke study (REGARDS) [4]. The study found that VTE related events occurred in 51,149 individuals and from those individuals 17,318 were black. The studies point out that when compared to whites blacks had a higher rate of VTE in CHS (HR 1.81; 95\% CI 1.20, 2.73). The reasons for Geographic and Racial Differences in Stroke study (REGARDS) 
pointed out that there was a significant region by race interaction $(\mathrm{p}=0.01)$. The study found that blacks in the southeast had a higher rate of VTE than blacks in the rest of the US (HR 1.63; 95\% CI 1.08, 2.48). These findings were not seen in whites (HR 0.83; 95\% CI 0.61, 1.14) [4].

Even though clinical data note that most cases of PE occur between 60 and 70 years of age, autopsy reports show the highest incidence of PE in individuals who are 70 to 80 years of age [3]. The actual figures might be even higher because silent PEs can develop in up to $40 \%$ to $50 \%$ of patients [3].

Belohlavek et al. [3] found that up to $10 \%$ of acute PE patients die suddenly, and most patients die within two hours of presentation.

\section{Pathophysiology}

Virchow triad (venous stasis, hypercoagulability states, and venous endothelial injury) is among the physiologic factors that contribute to venous thrombosis and consequently VTE. Also, thrombophilias, such as protein $\mathrm{C}$ and $\mathrm{S}$ deficiency, factor V Leiden mutation, and antithrombin III deficiency, are inherited disorders that also affect coagulation status and contribute to the development of VTE [1]. In addition, VTE is related to an individual patient's risk factors and circumstances regarding where it occurs. Prolonged bed rest, surgery, obesity, use of oral contraceptives, fractures, trauma, childbirth, spinal cord injury, and heart disease contribute to the development of VTE [3]. These triggering factors lead to endothelial cell activation, hypercoagulability, and stasis.

Increased use of oral contraceptives and hormone replacement therapy are linked to an increase in resistance to endogenous anticoagulants [2] [17]. Studies show that oral estrogens increase the risk of VTE complications depending on their type and dosage [5]. Bińkowska [5] found that dydrogesterone and micronized progesterone have no effect on the risk of VTE, but nor pregnane progestagens cause an additional increase in risk.

In 2003, The British Thoracic Society classified PE risk factors into major and minor categories and included and considered critically ill patients receiving intensive care to be at risk for developing VTE. In fact, Minet et al. [6] found that critically ill patients have a higher risk of VTE since these patients have added risk factors of VTE, such as sedation, immobilization, vasopressors, or central venous catheters. Some examples of major risk factors include: surgery, late pregnancy, caesarian section, fractures, varicose veins malignancy, reduced mobility, major abdominal/pelvic surgery, postoperative intensive care, advanced/metastatic cancer, hospitalization, and previous VTE. On the other hand, minor risk factors include: congenital heart disease, congestive heart failure, hypertension, indwelling central vein catheter, oral contraceptives, hormone replacement therapy, long distance sedentary travel, and obesity [3].

Goldman and Schafer [1] state that in at least $90 \%$ of patients, PE originates from a DVT in the lower legs and that approximately $70 \%$ of patients with proven PE still have DVT on presentation. The lower lobes of the lungs are af- 
fected more frequently than the upper lobes even though PE often involves both lungs. However, underlying diseases and comorbidities have more influence on prognosis than the extent and size of disease [7].

Moreover, the clinical impact of $\mathrm{PE}$ is reliant on the time frame over which vascular obstruction occurred, the extent of reduction in pulmonary blood flow, and the presence or absence of underlying cardiopulmonary disease [1]. Also, Goldman and Schafer [1] argue that obstruction of the pulmonary arteries contributes to hypoxemia in PE. An increased alveolar-arterial oxygen tension gradient reflects inadequate oxygen transfer across the lungs due to the alveolar dead space that occurs as a result of PE. Therefore, for patients who are elderly, debilitated, frail, or have cardiac or pulmonary disease, even a small obstruction in the pulmonary circulation could lead to pulmonary hypertension, after load increase, and RV dilation [3].

\section{Clinical Manifestations}

The clinical manifestations of PE are dependent on the location and size of the obstruction [6]. For example, healthy patients presenting with a small PE may have stable vital signs and be asymptomatic, whereas patients with significant comorbidities may become unstable with even a small PE [8].

Most patients with PE present with chest pain, dyspnea, or increased respiratory rate, and more than $85 \%$ of patients with $\mathrm{PE}$ tend to present with sudden onset or progressive exercise-induced dyspnea [3]. Additionally, more than 50\% of patients also present with chest pain that might be difficult to distinguish from angina of ischemic origin since the pain is sharp and worse with breathing [8]. In general, the nurse practitioner should consider a diagnosis of PE in a patient presenting with dyspnea, chest pain, syncope, or hemoptysis, especially in patients who are also positive for DVT [8]. However, the triad of symptoms most often reported with PE is dyspnea, chest pain, or hemoptysis [1].

Other clinical manifestations of PE might include a cough in more than $20 \%$ cases, hemoptysis in more than $7 \%$, and syncope in more than $14 \%$ of patients [3].

Lastly, there are variations of subjective and objective findings in PE. Subjective findings include sudden onset dyspnea, substernal discomfort, pleuritic chest pain, palpitations, anxiety, sense of impending doom, or lightheadedness. On the other hand, objective findings include hemoptysis, tachypnea, tachycardia, hypoxemia, initially elevated blood pressure, diaphoresis, decreased cardiac output, hypotension, shock, or signs of RV overload [9].

\section{Diagnosis and Diagnostic Tests}

Most patients presenting with PE will have one or more of the following clinical features: sudden onset dyspnea, substernal/pleuritic chest discomfort, or tachypnea.

Therefore, the nurse practitioner must consider the following differential di- 
agnosis when formulating a treatment plan: pneumonia, chronic obstructive pulmonary disease exacerbation, acute respiratory distress syndrome, acute bronchitis, aspiration, congestive heart failure, acute coronary syndrome, pericarditis, pneumothorax, rib fracture, or anxiety [17].

PE diagnosis warrants objective testing since the clinical manifestations are nonspecific and could lead to misdiagnosis or underdiagnosis. Moreover, the nurse practitioner should use a validated clinical prediction rule such the Wells or Geneva Rules to choose based on a patient's risk for PE.

The Wells Clinical Prediction Rule includes the presence of risk factors for VTE, clinical assessment components, and the absence of an alternative diagnosis. Based on the Wells Clinical Prediction Rule for Likelihood of Pulmonary Embolism, the likelihood of PE is classified into low $(<2)$, moderate $(2-6)$, or high (>6) probability of PE [1]. This tool is significant because it gives the clinician a guide for further testing, such as D-dimer assay, and noninvasive testing, such as computed tomography (CT), pulmonary angiography or ventilation-perfusion lung scan (VQ scan) [10]. According to Goldman and Schafer [1], these last two tests have replaced pulmonary angiography for PE diagnosis.

In 2015, Raja et al. [11] encouraged the practitioner not to obtain D-dimer measurements or imaging studies with a low pretest probability of PE. The study also urged the practitioner to order a $\mathrm{D}$-dimer as the initial diagnostic test in patients who have an intermediate pretest probability and to avoid ordering a $\mathrm{D}$-dimer assay if a high pretest probability of PE exists [11]. Studies show that a $\mathrm{D}$-dimer level is an indirect measure of ongoing coagulation system activation, and it is a helpful test to avoid further diagnostic testing when there is a low $(<2)$ or moderate (2 - 6) pretest likelihood of PE [1] [11]. However, current research shows that a D-dimer assay has $85 \%$ to $98 \%$ sensitivity for PE diagnosis but has a low specificity [11]. Therefore, a D-dimer assay is indicated if there is low to moderate pretest likelihood of PE. A negative D-dimer helps exclude a PE diagnosis, whereas a positive test requires further testing via CT pulmonary angiography. If the patient presents with a high pretest likelihood of PE, the nurse practitioner should consider first testing with CT pulmonary angiography [1] [17].

A chest radiograph and electrocardiogram (ECG) are also ordered since these tests are cost-effective and help the practitioner rule out pneumonia, pneumothorax, rib fracture, or acute coronary syndrome. Since emboli can cause right-sided heart strain and increased pulmonary vascular congestion, an ECG is used to assess signs of right heart strain [2]. Even though an ECG is not diagnostic, this test is helpful in ruling out acute coronary syndrome (ACS) when PE is suspected. Between $20 \%$ - 25\% of patients may present with a normal ECG, whereas up to $70 \%$ of patients may have ECG changes that are also seen in ACS [8]. The most common abnormal findings are sinus tachycardia, the classic pattern of S1Q3T3, complete or incomplete right bundle branch block, or T-wave inversions seen in the precordial leads. 
An arterial blood gas (ABG) analysis measures the levels of oxygen and carbon dioxide in the blood. ABG analysis might show acute respiratory alkalosis and a variable degree of hypoxemia.

Venous Doppler studies may reveal the presence of DVT, whereas a high-resolution CT scan of the chest reliably shows central PE [9].

Diagnostic tests, which include D-dimer measurement, Doppler ultrasound, chest CT pulmonary angiography, or V/Q lung scan, are safe and cost-effective and have replaced the traditional gold standard diagnostic tests of venography and pulmonary angiography [12]. Currently, ventilation/perfusion lung scan and pulmonary angiography tests are considered the gold standard for PE diagnosis [1].

Even though the practitioner would prefer a diagnostic test that will give a "black or white" result, the reality is more complicated. Since there is no single and perfectly accurate diagnostic test, research shows that several diagnostic tests should be combined for accuracy and cost-effectiveness [12]. However, concerns arise because of potential over-diagnosis of VTE with the new technology.

Goldman and Schafer [1] postulate that CT pulmonary angiography replaced VQ scans when diagnosing PE because it provides quick results and better visualization of thrombi in the pulmonary arteries. However, VQ lung scan continues to be the diagnostic test of choice when patients have kidney injury, are allergic to contrast media, or are women under 40 years old. Moreover, when CT pulmonary angiography is combined with $\mathrm{CT}$ venography in a single test for the diagnosis of PE and DVT, there is a lower risk of contrast dye injury with $90 \%$ sensitivity as compared to $83 \%$ sensitivity when CT pulmonary angiography is performed alone [1]. However, the nurse practitioner must take into consideration that $\mathrm{CT}$ venography poses an added risk of radiation exposure. Therefore, venous compression ultrasound is preferred when compared with CT venography because there is no risk of ionizing radiation exposure [1].

Even though gadolinium-enhanced magnetic resonance imaging (MRI) does not subject the patient to ionizing radiation, there has been data that show gadolinium-induced nephrogenic systemic fibrosis [13]. Gadolinium contrast medium is used in approximately 1 in 3 of MRI scans to improve the clarity of the pictures, but this test should be used only for patients for whom standard testing is contraindicated [1].

Additionally, the nurse practitioner should order the following additional tests to aid with PE diagnosis: complete blood count $(\mathrm{CBC})$, basic metabolic panel (BMP), liver panel, activated partial thromboplastin time (aPTT), international normalized ratio (INR), and brain natriuretic peptide (BNP).

New challenges arise currently due to concerns about potential VTE overdiagnosis and a lower clinical suspicion threshold [12]. Additionally, further research is needed for patients with prior VTE, pregnant women, or elderly patients since diagnostic management remains suboptimal in these populations [12]. 


\section{Management}

PE is a potentially life-threatening disease that can be treated medically, surgically, or percutaneously. The initial approach to patients with PE depends upon whether the patient is hemodynamically stable or unstable. Treatment goals should focus on the prevention of DVT and thromboembolic disease development.

The Food and Drug Administration (FDA) has approved the following treatments for PE: systemic thrombolysis, oral anticoagulants, Greenfield suction embolectomy catheter, and catheter-directed thrombolysis via ultrasound [14].

Currently, anticoagulation therapy is the cornerstone of PE treatment and should be initiated immediately with a rapid-acting parenteral anticoagulant, such as heparin, low-molecular-weight heparin (LMWH), fondaparinux, or a direct orally active factor Xa inhibitor. Additionally, Warfarin, an oral anticoagulant, has been successfully used in patients who require long-term treatment due to a high risk of thromboembolic development [1].

Current guidelines show that reperfusion therapy with fibrinolytic therapy is preferably started within 48 hours of the onset of symptoms and is selected based on absolute vs. relative contraindications. Moreover, thrombolytic therapy is indicated for hemodynamically unstable patients with a massive acute PE. However, despite its potential benefit, thrombolytic treatment can cause significant bleeding complications [15]. As a result, systemic thrombolysis is often not recommended due to the risk of intracranial bleeding with a 30-day mortality of $25 \%-65 \%$ [1]. The decision to administer thrombolysis is strongly influenced by additional clinical factors, such as PE-induced shock, unconsciousness, or high doses of pressors [15].

If a high risk exists with fibrinolytic therapy or if this treatment has failed for the patient, surgical pulmonary embolectomy is considered for severe PE, such as saddle embolus [1]. According to Zarghouni et al. [14], there has been increased clinical interest in catheter-directed interventions (CDI) since there is high morbidity and mortality associated with surgical embolectomy. Therefore, mechanical reperfusion with percutaneous catheter embolectomy with thrombus fragmentation or catheter-directed fibrinolytic therapy is valid options for PE treatment [1]. Currently, percutaneous mechanical thrombectomy represents an appealing option because it rapidly reduces the clot and strain on the right heart while reestablishing adequate circulation [1].

AngioJet and Hydrolyzer are thrombectomy systems, but they both have been associated with bradyarrhythmias when used in the pulmonary arteries. Moreover, 10 F Pronto .035" and 14 F XL extraction catheter (Vascular Solutions, Minneapolis, MN) are being investigated for the treatment of acute symptomatic PE since they are easier to manipulate over a wire, offering protection from pulmonary artery wall perforation [16]. Since 2013, Heberlein et al. [16] postulated that the Pronto .035" and XL extraction catheter should be considered in acute symptomatic PE since they are fast, inexpensive, highly maneuverable, and 
efficient in thrombus removal. However, a deeper understanding of next generation aspiration catheters could provide novel insights for treating acute symptomatic PE.

Lastly, permanent or retrievable filters in the inferior vena cava reduce the risk of recurrent $\mathrm{PE}$ and are indicated with an absolute risk of anticoagulation and when there is a high risk of further emboli [2].

Interest in catheter-directed techniques has increased considerably because anticoagulation alone often fails to promptly improve right ventricular strain and systemic thrombolysis and surgical thrombectomy have high morbidity and mortality. According to Zarghouni et al. [14], CDI are seen more frequently in inpatient treatment, and recently, the FDA approved ultrasound-accelerated catheter-directed thrombolysis for PE treatment.

The treatment of PE, however, continues to be a challenge because of acute onset right ventricular strain with right-sided heart failure, sudden death, pulmonary infarction, or cardiogenic shock, limiting the time for therapeutic success. Thus, when feasible, the nurse practitioner should adopt a multidisciplinary approach to facilitate management of hemodynamically unstable patients with PE while considering that some centers have incorporated a "pulmonary embolism response team" (PERT) to facilitate the process [15].

\section{Prevention: Patient and Staff Education}

$\mathrm{PE}$ is the most common preventable cause of death in hospitalized patients in the United States [2]. At least half of the patients with newly diagnosed VTE have a history of recent hospitalization, and most patients failed to receive thromboprophylaxis during their hospital stay. Therefore, prevention should focus on identifying people at risk, minimizing venous stasis, and early diagnosis. Educating patients about early mobility after surgery is crucial for prevention of PE and even death. For at risk individuals, the prevention of venous stasis with graded compression stockings or graded compression boots is indicated.

In 2015, Haxaire, Tromeur, Couturaud, \& Leroyer found that an increased level of anxiety exists at and after PE diagnosis is made and that most patients underestimate the potential life-threatening episode. In fact, the study notes that there is a gap between perceptions and current medical knowledge regarding VTE. Therefore, the practitioner must gather more information on the attitudes of the VTE episode and its consequences as part of any health education process [18].

\section{Prognosis}

With adequate anticoagulant therapy, most patients with PE survive, and the case fatality rates at 1 month after diagnosis of DVT or PE are up to 6\%-12\% [1].

Flinterman et al. examined the long-term survival rate in a large cohort of 4947 patients, aged 18 to 70 years, with first nonfatal VTE or PE over a period of 
8 years [19]. The study found that the overall mortality rate was 22.7 per 1000 person-years (95\% CI 21.0 - 24.6) for patients and 4.7 per 1000 person-years (95\% CI 4.0 - 5.6) for controls [19]. Also, Flinterman et al. found that patients with VTE had a 4.0-fold (95\% CI 3.7 - 4.3) risk of death compared with the control group. The highest risk of death was seen in patients who also presented with malignancies, diseases of the circulatory system, and venous thrombosis [19].

\section{Conclusions}

Recently, growth in the availability and use of CT pulmonary angiography (CTPA) when evaluating patients with suspected PEs has contributed to a new trend in the healthcare system. The literature shows that despite an increase in the number of PEs, mortality has remained unchanged and that PE might in fact be overdiagnosed. Therefore, limiting CT scan use is encouraged since there is an increased risk of radiation, contrast-induced nephropathy, or contrast allergy [1].

Further studies are needed to collect data to evaluate the safety and net clinical benefit of this approach. In fact, a new formal cost-effectiveness study might help establish whether changing the current diagnostic strategy will result in better symptomatic management of suspected acute PE [18].

\section{Acknowledgements}

The authors have no conflicts of interest that are relevant to the content of this article.

\section{References}

[1] Goldman, L. and Schafer, A.I. (2015) Goldman-Cecil Medicine. Elsevier Saunders, Philadelphia, PA.

[2] Grossman, S. and Porth, M.C. (2014) Porth's Pathophysiology: Concepts of Altered Health States. Lippincott Williams \& Wilkins, Philadelphia, PA.

[3] Belohlavek, J., Dytrych, V. and Linhart, A. (2013) Pulmonary Embolism, Part I: Epidemiology, Risk Factors and Risk Stratification, Pathophysiology, Clinical Presentation, Diagnosis and Nonthrombotic Pulmonary Embolism. Experimental and Clinical Cardiology, 18, 129-138.

[4] Zakai, N.A., McClure, L.A., Judd, S.E., Safford, M.M., Folsom, A.R., Lutsey, P.L. and Cushman, M. (2014) Racial and Regional Differences in Venous Thromboembolism in the United States in 3 Cohorts. Circulation, 129, 1502-1509. https://doi.org/10.1161/CIRCULATIONAHA.113.006472

[5] Bińkowska, M. (2014) Menopausal Hormone Therapy and Venous Thromboembolism. Menopause Review, 13, 267-272. https://doi.org/10.5114/pm.2014.46468

[6] Minet, C., Potton, L., Bonadona, A., Hamidfar-Roy, R., Somohano, C.A., Lugosi, M., Cartier, J.-C., Ferretti, G., Schwebel, C. and Timsit, J.-F. (2015) Venous Thromboembolism in the ICU: Main Characteristics, Diagnosis and Thromboprophylaxis. Critical Care, 19, 287. https://doi.org/10.1186/s13054-015-1003-9

[7] Garcia-Sanz, M., Pena-Alvarez, C., Lopez-Landeiro, P., Bermo-Dominguez, A., 
Fonturbel, T. and Barcala, F.J.G. (2014) Symptoms, Location and Prognosis of Pulmonary Embolism. Revista Portuguesa de Pneumologia, 20, 194-199. https://doi.org/10.1016/j.rppneu.2013.09.006

[8] Brown, A., Dingle, H.E., Brywczynski, J., McKinney, J.J. and Slovis, C.M. (2017) The 5 Deadly Causes of Chest Pain: Recognizing the Potentially Lethal Causes Other than Myocardial Infarction. A Journal of Emergency Medical Services, 42, 55-63.

[9] Barkley, T.W. and Myers, C.M. (2015) Practice Considerations for Adult-Gerontology Acute Care Nurse Practitioners. Barkley \& Associates, West Hollywood, CA.

[10] Modi, S., Deisler, R., Gozel, K., Reicks, P., Irwin, E., Brunsvold, M., Banton, K. and Beilman, G.J. (2016) Wells Criteria for DVT Is a Reliable Clinical Tool To Assess the Risk of Deep Venous Thrombosis in Trauma Patients. World Journal of Emergency Surgery, 11, 24. https://doi.org/10.1186/s13017-016-0078-1

[11] Raja, A.S., Greenberg, J.O., Qaseem, A., et al. (2015) Evaluation of Patients with Suspected Acute Pulmonary Embolism: Best Practice Advice from the Clinical Guidelines Committee of the American College of Physicians. Annals of Internal Medicine, 163, 701-711. https://doi.org/10.7326/M14-1772

[12] Le Gal, G. and Righini, M. (2015) Controversies in the Diagnosis of Venous Thromboembolism. Journal of Thrombosis and Haemostasis, 13, S259-S265.

[13] Ferris, N. and Goergen, S. (2017) Gadolinium Contrast Medium (MRI Contrast Agents). Inside Radiology, General Radiology.

https://www.insideradiology.com.au/gadolinium-contrast-medium/

[14] Zarghouni, M., Charles, H.W., Maldonado, T.S. and Deipolyi, A.R. (2016) Catheter-Directed Interventions for Pulmonary Embolism. Cardiovascular Diagnosis and Therapy, 6, 651-661. https://doi.org/10.21037/cdt.2016.11.15

[15] Tapson, V.F. (2008) Acute Pulmonary Embolism. The New England Journal of Medicine, 358, 1037-1052. https://doi.org/10.1056/NEJMra072753

[16] Heberlein, W.E., Meek, M.E., Saleh, O., Meek, J.C., Lensing, S.Y. and Culp, W.C. (2013) New Generation Aspiration Catheter: Feasibility in the Treatment of Pulmonary Embolism. World Journal of Radiology, 5, 430-435. https://doi.org/10.4329/wjr.v5.i11.430

[17] Hamilton-Craig, C.R., McNeil, K., Dunning, J., Walters, D.L., Slaughter, R. and Kermeen, F. (2008) Treatment Options and Strategies for Acute Severe Pulmonary Embolism. Internal Medicine Journal, 38, 657-667. http://onlinelibrary.wiley.com/doi/10.1111/j.1445-5994.2008.01671.x/abstract https://doi.org/10.1111/j.1445-5994.2008.01671.x

[18] Haxaire, C., Tromeur, C., Couturaud, F. and Leroyer, C. (2015) A Qualitative Study to Appraise Patients and Family Members Perceptions, Knowledge, and Attitudes towards Venous Thromboembolism Risk. PLOS ONE, 10, e0142070. https://doi.org/10.1371/journal.pone.0142070

[19] Flinterman, L.E., Vlieg, A.V., Cannegieter, S.C. and Rosendaal, F.R. (2012) Long-Term Survival in a Large Cohort of Patients with Venous Thrombosis: Incidence and Predictors. PLoS Medicine, 9, e1001155.

http://journals.plos.org/plosmedicine/article?id=10.1371\%2Fjournal.pmed.1001155 https://doi.org/10.1371/journal.pmed.1001155 